\title{
Polymorphisms of Ionotropic Glutamate Receptor-Related Genes and the Risk of Autism Spectrum Disorder in a Chinese Population
}

\author{
Xinyan Xie ${ }^{1 *}$, Fang Hou'* ${ }^{1 *} \mathrm{Li}^{2}$, Yanlin Chen², Lingfei Liu1, Xiu Luo', \\ Huaiting $\mathrm{Gu}^{1}$, Xin $\mathrm{Li}^{1}$, Jiajia Zhang ${ }^{3}$, Jianhua Gong ${ }^{2} \otimes$, and Ranran Song ${ }^{1} \bowtie$ \\ 1'Department of Maternal and Child Health and MOE Key Lab of Environment and Health, School of Public Health, Tongji Medical College, \\ Huazhong University of Science and Technology, Wuhan, China \\ ${ }^{2}$ Maternity and Children Health Care Hospital of Luohu District, Shenzhen, China \\ ${ }^{3}$ Department of Epidemiology and Biostatistics, Arnold School of Public Health, University of South Carolina, Columbia, USA
}

Objective To evaluate the association of GRIK2 and NLGN1 with autism spectrum disorder in a Chinese population.

Methods We performed spatio-temporal expression analysis of GRIK2 and NLGN1 in the developing prefrontal cortex, and examined the expression of the genes in ASD cases and healthy controls using the GSE38322 data set. Following, we performed a case-control study in a Chinese population.

Results The analysis using the publicly available expression data showed that GRIK2 and NLGN1 may have a role in the development of human brain and contribute to the risk of ASD. Later genetic analysis in the Chinese population showed that the GRIK2 rs6922753 for the $\mathrm{T}$ allele, TC genotype and dominant model played a significant protective role in ASD susceptibility (respectively: $\mathrm{OR}=0.840$, $\mathrm{p}=0.023$; $\mathrm{OR}=0.802, \mathrm{p}=0.038 ; \mathrm{OR}=0.791, \mathrm{p}=0.020$ ). The $N L G N 1 \mathrm{rs} 9855544$ for the $\mathrm{G}$ allele and GG genotype played a significant protective role in ASD susceptibility (respectively: $\mathrm{OR}=0.844, \mathrm{p}=0.019 ; \mathrm{OR}=0.717, \mathrm{p}=0.022$ ). After adjusting $\mathrm{p}$ values, the statistical significance was lost ( $\mathrm{p}>0.05)$.

Conclusion Our results suggested that GRIK2 rs6922753 and NLGN1 rs9855544 might not confer susceptibility to ASD in the Chinese population.

Psychiatry Investig 2019;16(5):379-385

Key Words Autism spectrum disorder, Ionotropic glutamate receptors, GRIK2, NLGN1, Polymorphism.

\section{INTRODUCTION}

Glutamate ionotropic receptor kainate type subunit 2 (GRIK2) and neuroligin-1 (NLGN1) could play a part in the function of the ionotropic glutamate receptors (iGluRs) and thus, influ-

\footnotetext{
Received: November 2, 2018 Revised: February 1, 2019

Accepted: February 26, 2019

$\triangle$ Correspondence: Ranran Song, PhD

Department of Maternal and Child Health and MOE Key Lab of Environment and Health, School of Public Health, Tongji Medical College, Huazhong University of Science and Technology, No 13 Hangkong Road, Wuhan, Hubei, China

Tel: +86-27-836-57509, Fax: +86-27-836-57509,

E-mail: songranran@hust.edu.cn
}

$\triangle$ Correspondence: Jianhua Gong, MD

Maternity and Children Health Care Hospital of Luohu District, No.2018 Taibai Road, Shenzhen, China

Tel: +86-0755-25800182, Fax: +86-0755-25800182,

E-mail: 768186112@qq.com

*These authors contributed equally to this work.

@ This is an Open Access article distributed under the terms of the Creative Commons Attribution Non-Commercial License (https://creativecommons.org/licenses/by$\mathrm{nc} / 4.0$ ) which permits unrestricted non-commercial use, distribution, and reproduction in any medium, provided the original work is properly cited. ence glutamate signaling and neuronal growth. ${ }^{1-4} N L G N 1$ are specific to excitatory synapses with the capacity to enhance excitatory synapses depending on $\mathrm{Ca}^{2+} /$ calmodulin kinase II (CaMKII), which robustly phosphorylates the T739 domain of NLGN1. ${ }^{1}$ While GRIK2 acts presynaptically to decrease glutamatergic transmission in the hippocampus. ${ }^{2}$ Animal studies showed that the GluK2 receptor, one of the kainite receptors encoded by the GRIK2 gene, regulates the maturation of synaptic circuits involved in learning and memory. ${ }^{5}$ Familybased association study in the Korean trios found preferential transmission of the $\mathrm{C}$ allele at the rs3213607 of GRIK2 in ASD. ${ }^{6}$ For the European population, there was also a family-based association study identifying the GRIK2 as an ASD candidate gene. ${ }^{7}$ However, the family-based association study, combined with case-control study, based on an Indian population failed to find an association between GRIK2 and ASD. ${ }^{8}$ A NLGN1 Pro89Leu (P89L) missense variant was found in ASD patients in the USA. Moreover, in knock-in P89L mice, the model mice showed abnormal social behavior. Previous studies showed 
altered expression of NLGN1 in the brain was observed in several mouse models for ASD, such as Fmr1 and Eif4ebp2 knockout mice. ${ }^{10,11}$ However, there is little research focusing on the association between ASD and GRIK2 and NLGN1 among the Chinese population. Based on the roles of GRIK2 and NLGN1 in iGluRs and their inconsistent results, we think it is worthwhile to verify the association between ASD and GRIK2 and NLGN1 in the Chinese Han population.

Accumulating evidence suggests that ASD is likely a neurodevelopmental disorder. ${ }^{12-14}$ Yuen et al. ${ }^{15}$ found 61 ASD-risk genes with sequence-level mutations, which were enriched in synaptic transmission, transcriptional regulation and RNA processing functions. The genes associated with transcriptional regulation and RNA processing are more often expressed in the brain prenatally, while synaptic-function-related genes are expressed in brain throughout development. Based on the neurodevelopmental hypothesis, if the GRIK2 and NLGN1 genes are involved in brain development they may be expressed in developing human brain and fluctuate with brain development. We, therefore, explored the spatio-temporal expression pattern in the developing prefrontal cortex using publicly available expression data from Brainspan. ${ }^{16}$ Furthermore, if the genes are true risk genes for ASD, the expression of the genes should be dysregulated in ASD. We obtained the publicly available expression data set GSE38322 ${ }^{17}$ and performed Student's t-test. Finally, in our case-control study, we investigated whether the GRIK2 and NLGN1 genes were associated with ASD risk in a Chinese population including 504 ASD patients and 1923 healthy controls.

\section{METHODS}

\section{Spatio-temporal expression pattern analysis of risk genes}

To explore the spatio-temporal expression of GRIK2 and NLGN1 genes in developing human brain, we downloaded the expression data (based on RNA sequencing) from the Allen Institute for Brain Science ${ }^{16}$ (http://www.brain-map.org/) (access date: 10/16/2016) ( $\mathrm{n}=42$ individuals). We divided the prefrontal cortex into four sub-regions, including dorsolateral prefrontal cortex (DFC), ventrolateral prefrontal cortex (VFC), medial prefrontal cortex (MFC) and orbital prefrontal cortex (OFC). ${ }^{18}$ The original expression values were linearly transformed using a min-max standardization method with the following function: $\mathrm{x}=(\mathrm{x}-\mathrm{min}) /(\max -\mathrm{min})$, where $\mathrm{x}$ represents the original expression value.

\section{Expression analysis in ASD cases and controls}

To explore whether GRIK2 and NLGN1 genes are differentially expressed in ASD cases compared with controls, we ob- tained the publicly available expression data set GSE38322. ${ }^{17}$ GSE38322 contains brain transcriptional [including cerebellum and occipital (BA19)] data of 18 ASD cases and 18 controls. We downloaded the raw expression values from GEO (https://www.ncbi.nlm.nih.gov/gds/) and performed Student's t-test.

\section{Experiment in Chinese population}

\section{Subjects}

Our study included 504 ASD patients and 1923 healthy controls. The ASD patients were recruited from the Maternal and Child Care Service Centre in Shenzhen city, Zhuhai city and Luohu district in China, Wuhan Mental Health Center in China and Special Children's Education Agency in Suzhou, Guangzhou and Wuhan in China between July 2010 and July 2016. ASD patients were diagnosed by professional neurologists based on the Diagnostic and Statistical Manual of Mental Disorders Fourth Edition (DSM-IV). The control data were selected from GWAS data for a healthy population without ASD, attention-deficit/hyperactivity disorder, mental retardation or other neurodevelopmental disorders in a Chinese population, and they were matched with ASD patients in gender. This case-control study was approved by the Ethics Committee of Tongji Medical College of Huazhong University of Science and Technology, China.

\section{Identification of Candidate SNPs and Genotyping}

The procedure for screening candidate SNPs that might be functional in GRIK2 gene and NLGN1 gene was as follows. First, we extracted the SNPs having possible functional effects of protein coding, splicing regulation, transcriptional regulation or post-translation from the F-SNP database (http://compbio.cs.queensu.ca/F-SNP). Second, these SNPs whose minor allele frequency (MAF) of Han Chinese in Beijing (CHB) are more than 5\% were filtered from the HapMap database (http:// hapmap.ncbi.nlm.nih.gov/). Third, we assessed the linkage disequilibrium (LD) among these SNPs using SNAP Pairwise (http://www.broadinstitute.org/mpg/snap/ldsearchpw.php); if the SNPs were in strong LD with each other $\left(r^{2} \geq 0.80\right)$, we considered reserving only one to analyze any further. As a result, there were two SNPs, which could be used to further selection, in GRIK2 gene (rs6922753) and NLGN1 gene (rs9855544).

Genomic DNA was extracted from oral swabs sample using TIANamp Swab DNA Kit DP080714 (Tiangen, Beijing, China) by reference to the manufacturer's instructions. DNA concentration and optical density were tested by a NanoDrop 1000 spectrophotometer (Thermo Fisher Scientific, Waltham, MA, USA). Genotyping was performed at the BIO MIAOBIOLOGICAL Corporation (Beijing, China) with the Seque- 
nomMassARRAY platform (San Diego, CA, USA) according to the manufacturer's protocol. The MassARRAY Assay Designer software (v3.1) was used to design PCR primers and termination mixes for multiplexed assays. The mass of extended primer was determined using a MALDI-TOF mass spectrometer and we analyzed the resulting genotype spectra using Mass ARRAY Type4.0 software.

\section{Statistical analysis}

The ggplot2 package (http://ggplot2.org/) in R (v3.2.5) was used to plot the spatial-temporal expression patterns of the risk genes. GEOquery (http://geoquery.org/) and ggplot2 package were used to analyze the expression pattern in ASD cases and controls from GED dataset. SPSS software v22.0 was used for statistical analyses in experiment of Chinese population. The Hardy-Weinberg equilibrium (HWE) for genotypes was analyzed by Goodness-of-fit $\chi^{2}$ test in the healthy controls. Unconditional logistic regression (LR) using dominant, recessive and genotype models for each SNP were executed in association analysis. Odds ratios (OR) and 95\% confidence intervals (95\% CI) were adopted to assess the relative risk conferred by a possibly risk allele and genotype. To control for the false discovery rate (FDR), the Benjamin-Hochberg method was used to adjust the $\mathrm{p}$ values for multiple tests within the univariate LR analysis. The statistical power to detect the effects of the SNPs was calculated by Power v3.0.0. For example, for SNPs with minor allele frequency (MAF) of 0.312 , and the prevalence of ASD in China was 2.00\%, the power of the sample size to detect an OR of 1.50 was $88.4 \%$. All p values were two-tailed with a statistical significant level set at 0.05 .

\section{Informed consent and confidentiality}

The experiments of the article was approved by the Ethics Committee of Tongji Medical College of Huazhong University of Science and Technology, China. Informed consent was acquired from the participants or participants' guardians. The patient's information was confidential. An ID was given to each participant. There were no real names, initials, or disclose information that might identify a particular person.

\section{Ethical approval}

All procedures performed in studies involving human participants were in accordance with the 1964 Helsinki declaration and its later amendments.

\section{RESULTS}

\section{Spatio-temporal expression pattern analysis of risk genes}

The NLGN1 gene showed higher expression levels at em- bryonic and fetal stages [8 pcw (post conception weeks) to 4 mos] compared with childhood and adulthood stages ( 8 yrs to $40 \mathrm{yrs}$ ) (Figure 1), suggesting this gene may have a role in neurodevelopment. ${ }^{19}$ The GRIK2 gene showed a trend of downregulated expression levels across age (Figure 1).

\section{Expression analysis in ASD cases and controls}

mRNA expression of NLGN1 in ASD was significantly upregulated in the cerebellum $(\mathrm{p}<0.001)$. There was no significant change in the occipital ( $\mathrm{p}=0.145$ ) (Figure 2). mRNA expression of GRIK2 did not show significant changes in the cerebellum ( $\mathrm{p}=0.088)$ or occipital $(\mathrm{p}=0.712)$ in the GSE38322 data set (Figure 2). Considering that the trend of GRIK2 expression level was downregulated across age in developing hu-

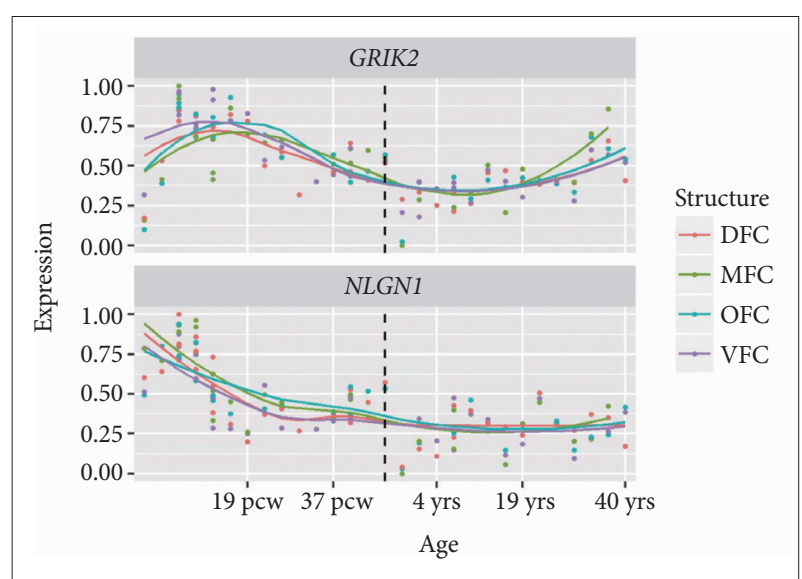

Figure 1. Expression patterns of the GRIK2, NLGN1 genes in human frontal cortex. Expression level of the genes across the entire developing stages [from 8 post-conception weeks ( $\mathrm{pcw}$ ) to 40 years (yrs)] were depicted in the frontal cortex, which was divided into DFC, MFC, OFC and VFC. The expression data were downloaded from Brainspan. DFC: dorsolateral prefrontal cortex, VFC: ventrolateral prefrontal cortex, MFC: medial prefrontal cortex, OFC: orbital prefrontal cortex.

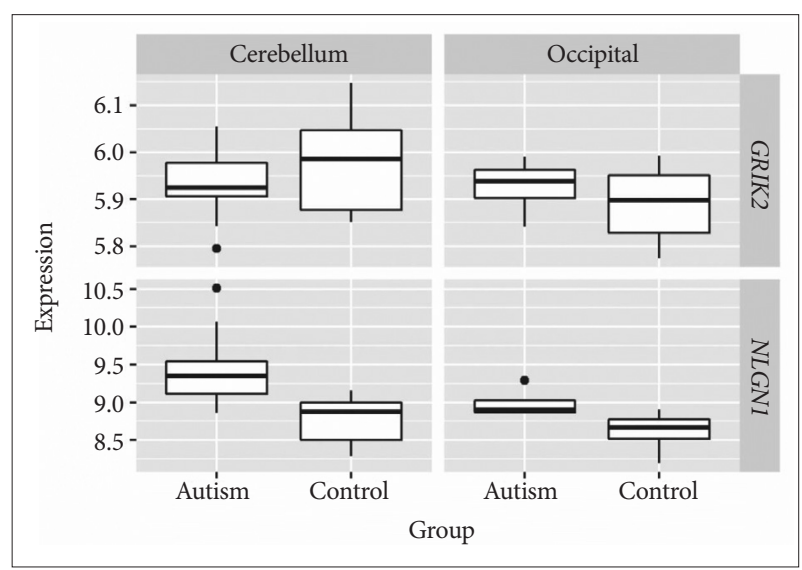

Figure 2. Dysregulation of GRIK2, NLGN1 genes in ASD cases vs cases controls. The vertical axis represented the mRNA expression of NLGN1 and GRIK2 genes in the cerebellum and occipital in GSE38322 data set. 
man brain from Brainspan ${ }^{16}$ and Webster's study showed the same trend in schizophrenia, ${ }^{20}$ we still included the GRIK2 gene in the subsequent validation study in the Chinese Han population.

\section{Experiment in Chinese population}

\section{Subjects' characteristics}

In this case-control study, there were 504 ASD patients (441 males and 63 females, $8.23 \pm 3.15$ years) and 1923 healthy controls (1683 males and 240 females, $61.38 \pm 8.51$ years) for analysis. There was no statistically significant difference in the distribution of gender $\left(\chi^{2}=0.000, p=0.991\right)$ between cases and controls. The ASD and controls were matched according gender (male:female ratio of 7:1).

\section{Association analysis between individual SNPs and ASD risk}

The two SNPs conformed to Hardy-Weinberg equilibrium ( $p>0.05$ ). The MAFs of the two SNPs were similar to those in the 1000 Genomes Project of Han Chinese in Beijing, China. The statistical power for detecting the effects of the SNPs were $88.4 \%$ and $90.8 \%$ (Table 1). As shown in Table 2, the two SNPs were significantly associated with ASD risk. The T allele and the TC genotype of the rs6922753 polymorphism in GRIK2 were significantly associated with decreased risk of ASD (respectively: $\mathrm{OR}=0.840,95 \% \mathrm{CI}=0.722-0.976, \mathrm{p}=0.023$; $\mathrm{OR}=$ $0.802,95 \% \mathrm{CI}=0.651-0.988, \mathrm{p}=0.038)$, as was the dominant model $(\mathrm{OR}=0.791,95 \% \mathrm{CI}=0.649-0.963, \mathrm{p}=0.020)$. The NLGN1 rs 9855544 polymorphism for the $G$ allele and GG genotype played a significant protective role in ASD susceptibility (respectively: $\mathrm{OR}=0.844,95 \% \mathrm{CI}=0.732-0.973, \mathrm{p}=0.019 ; \mathrm{OR}=0.717$, $95 \% \mathrm{CI}=0.539-0.954, \mathrm{p}=0.022$ ). However, after adjusting $\mathrm{p}$ values, the statistical significance was lost ( $\mathrm{p}>0.05)$.

\section{DISCUSSION}

In our study, we explored the spatio-temporal expression pattern in the developing prefrontal cortex and mRNA expression in ASD cases compared with controls of GRIK2 and NLGN1 genes using the publicly available expression data. The results suggested that the genes may have a role in the human brain and contribute to the risk of ASD. ${ }^{19}$ In the subsequent validation study in the Chinese Han population, we found that rs6922753 in GRIK2 and NLGN1 rs9855544 polymorphisms were unlikely to be associated with ASD. The sta-

Table 1. Basic information of SNPs in study

\begin{tabular}{cccccccc}
\hline \multicolumn{1}{c}{ Gene } & SNP & MA & MAF* $^{*}$ & MAF $^{\dagger}$ & MAF $^{\ddagger}$ & $p^{\S}$ & Power $(\%)$ \\
\hline GRIK2/GLUR6 & rs6922753 & T & 0.342 & 0.312 & 0.385 & 0.851 & 88.4 \\
NLGN1 & rs9855544 & G & 0.458 & 0.418 & 0.462 & 0.082 & 90.8 \\
\hline
\end{tabular}

*the minimum allele frequency of the SNPs in the control group, the minimum allele frequency of the SNPs in the 1000 Genomes Project in the Han Chinese in Beijing, China, \#the minimum allele frequency of the SNPs in the 1000 Genomes Project in the Japanese in Tokyo, Japan, $\S$ Hardy-Weinberg equilibrium test. MA: minor allele, MAF: minor allele frequency

Table 2. Association analysis between individual SNP and ASD risk

\begin{tabular}{|c|c|c|c|c|c|c|}
\hline SNP & Genotype & Case (\%) & Control (\%) & OR $(95 \% \mathrm{CI})$ & $\mathrm{p}^{*}$ & FDR-P \\
\hline \multirow[t]{7}{*}{ rs6922753 } & $\mathrm{C}$ & $696(69.6)$ & $2530(65.8)$ & 1.00 & & \\
\hline & $\mathrm{T}$ & $304(30.4)$ & $1316(34.2)$ & $0.840(0.722-0.976)$ & 0.023 & 0.058 \\
\hline & $\mathrm{CC}$ & $246(49.2)$ & $834(43.4)$ & 1.00 & & \\
\hline & TC & $204(40.8)$ & $862(44.8)$ & $0.802(0.651-0.988)$ & 0.038 & 0.076 \\
\hline & $\mathrm{TT}$ & $50(10.0)$ & $227(11.8)$ & $0.747(0.533-1.046)$ & 0.090 & 0.113 \\
\hline & Dominant model & & & $0.791(0.649-0.963)$ & 0.020 & 0.100 \\
\hline & Recessive model & & & $0.830(0.601-1.147)$ & 0.259 & 0.259 \\
\hline \multirow[t]{7}{*}{ rs9855544 } & A & $574(58.3)$ & $2083(54.2)$ & 1.00 & & \\
\hline & G & $410(41.7)$ & $1763(45.8)$ & $0.844(0.732-0.973)$ & 0.019 & 0.190 \\
\hline & $\mathrm{AA}$ & $171(34.8)$ & $583(30.3)$ & 1.00 & & \\
\hline & GA & $232(47.2)$ & $917(47.7)$ & $0.863(0.690-1.078)$ & 0.194 & 0.216 \\
\hline & GG & $89(18.1)$ & $423(22.0)$ & $0.717(0.539-0.954)$ & 0.022 & 0.073 \\
\hline & Dominant model & & & $0.817(0.662-1.007)$ & 0.058 & 0.097 \\
\hline & Recessive model & & & $0.783(0.608-1.009)$ & 0.059 & 0.084 \\
\hline
\end{tabular}

${ }^{*} \mathrm{p}$ values were calculated by binary logistic regression, adjusted by gender. OR: odds ratio, CI: confidence interval, FDR: false discovery rate 
tistical significance was lost after controlling for the false discovery rate (FDR). Studies with larger sample sizes are needed.

Synaptic transmission underlies every aspect of brain function. Excitatory synapses, which release the neurotransmitter glutamate, are the most numerous type of synapse in the brain. Furthermore, the trafficking of glutamate receptors to and from these synapses controls the strength of excitatory synaptic transmission. ${ }^{21}$ Glutamate receptors and synapses are shown to be related to disrupted synapse development and homeostasis in ASD. iGluRs are integral membrane proteins composed of four large subunits that form a central ion channel pore. Disruption in synaptic transmission often implicates reduced AMPA- and NMDA-dependent glutamatergic transmission. ${ }^{22}$ Weak NMDA antagonists may be effective in treating ASD, predicting that NMDA hyperfunction has a role in ASD. ${ }^{23}$ Downregulation of AMPARs is related to the reduction of plasticity and post-synaptic excitatory potentials, which are strongly associated with learning and memory, and disruption of these may underlie intellectual disabilities in ASD.22

The GRIK2 gene codes for the kainate receptor subunit 2. It has been suggested as a candidate gene for ASD because of its localization in the autism specific region on chromosome $6 q 21$ and the involvement of the receptor protein in cognitive functions like learning and memory. ${ }^{8,24}$ Recently, a chromosomal microarray (CMA) analysis describing a 19-year old patient showed two de novo microdeletions that spanned 10 genes including GRIK2. ${ }^{25}$ Mutation screening revealed several SNPs, including one nucleotide variation changing the protein (M867I) of GRIK2, which may be functionally relevant to the development of ASD. ${ }^{26}$ Shuang's family-based association study in Chinese Han trios demonstrated that GRIK2 rs2227281 and rs2227283 showed preferential transmission and revealed an association between the GluR6 locus and ASD. ${ }^{27}$ However, our study suggests that the rs6922753 in GRIK2 is unlikely to confer susceptibility to ASD in the Chinese population. Studies using larger samples, which are representative of all of the Chinese population, are needed. In addition, our study results are consistent with family-based studies in the Indian population, which also failed to show evidence of genetic association of GRIK2 with ASD. ${ }^{8}$

Neuroligins are postsynaptic cell adhesion molecules that are important for synaptic function. NLGN1 is localized predominantly to excitatory synapses and plays a pivotal role in brain glutamatergic transmission and cognition. ${ }^{1}$ NMDARs are dispensable for synapse formation and connect to NLGN1 in that both bind to PSD-95, which is proven to be associated with ASD in a previous study. ${ }^{28}$ NLGN1-mediated synaptic potentiation is diminished after chronic blockade of NMDARs, suggesting that the function of NLGN1 is influenced by NMDAR signaling. ${ }^{29}$ Previous studies have indicated that NLGN1 is associated with ASD, ${ }^{9}$ schizophrenia, ${ }^{30}$ Alzheimer's disease, ${ }^{31}$ depression $^{32}$ and post-traumatic stress disorder. ${ }^{33}$ A novel NLGN1 Pro89Leu (P89L) missense variant was found in two ASD siblings, which led to the impairment of spine formation and changes in protein degradation and cellular localization. The results were validated in an experiment of knock-in P89L mice. ${ }^{9}$ Although it has been reported in previous studies, we found the significance was lost between NLGN1 and ASD after FDR. One of the reasons for this discrepancy may be the heterogeneity of ASD and the difference between Chinese and other races. Our inability to detect an association with the SNPs does not imply that NLGN1 is not a candidate gene for ASD in the Chinese population. It is necessary to explore the possibility that other polymorphic variants of NLGN1 gene act as risk alleles for ASD before coming to a conclusion on its involvement in ASD.

To the best of our knowledge, this is the first study to explore a correlation between polymorphisms in these two iGluRs-related genes and ASD in a Chinese Han population combined with the analysis of publicly available datasets. We got the negative findings about the two SNPs. As we known, the ASD is a polygenic complex disease. Genome-wide association studies have identified some important single nucleotide polymorphisms, but none has a large enough effect to be deemed causal..$^{34}$ However, up to $40 \%$ of simplex families and $60 \%$ of multiplex families (in which more than one individual has autism) could have several single nucleotide polymorphisms that, when combined, have an additive effect on risk. ${ }^{35}$ These results demonstrate that a myriad of common variants of very small effect impacts ASD liability. In our study, the logistic regression showed the significant $\mathrm{p}$ value before FDR, which may show the minor effect of the two genes. The limitations of this study were that we did not exactly match our controls to cases. We used public controls from an exon chip genotyping database for a healthy population. The studies were based on the hypothesis that human genotypes generally do not change with age, and the differences that come from age could be diluted by the large sample size of controls. For another, there are many case-control studies in genetic studies. ${ }^{36-39}$ The common application of case-control research proves its rationality to some extent. However, we could not ignore the advantages of other study designs, such as family-based and twin studies, as the ASD is high familial. Further analyses based on rigorous case-control studies or family-based studies along with substantially larger sample size are required.

In summary, the analysis of the publicly available datasets indicated that GRIK2 and NLGN1 may have a role in the development of human brain and NLGN1 gene was dysregulated in the cerebellum of ASD cases. The following experiment in the Chinese Han population implied that the single poly- 
morphisms of rs6922753 in GRIK2 and rs9855544 in NLGN1 were unlikely to demonstrate an association with the development of ASD.

\section{Acknowledgments}

This work was supported by the National Natural Science Foundation of China (81872636) to S.R. and Deep Science and Technology Innovation Fund from Shenzhen (JCYJ20160428095110571). The authors are very grateful to all participants.

\section{Conflicts of Interest}

The authors have no potential conflicts of interest to disclose.

\section{Author Contributions}

Designed the experiments: Xinyan Xie, Fang Hou, Ranran Song, Jianhua Gong, Li Li. Performed the experiments: Xinyan Xie, Fang Hou, Huaiting $\mathrm{Gu}$, Lingfei Liu, Xiu Luo, Xin Li. Analyzed the data: Xinyan Xie, Fang Hou, Jiajia Zhang. Contributed the materials: Li Li, Yanlin Chen, Jianhua Gong. Writing — original draft: Xinyan Xie, Fang Hou. Writing — review \& editing: Ranran Song, Jianhua Gong.

\section{ORCID iDs}

\section{Ranran Song}

Jianhua Gong

Xinyan Xie

Fang Hou https://orcid.org/0000-0002-8354-7698 https://orcid.org/0000-0002-2577-1309 https://orcid.org/0000-0002-4174-188X https://orcid.org/0000-0002-4513-1552

\section{REFERENCES}

1. Bemben MA, Shipman SL, Hirai T, Herring BE, Li Y, Badger JD 2nd, et al. CaMKII phosphorylation of neuroligin-1 regulates excitatory synapses. Nat Neurosci 2014;17:56-64.

2. Meldrum BS. Glutamate as a neurotransmitter in the brain: review of physiology and pathology. J Nutr 2000;130(4S Suppl):1007S-1015S.

3. Sampaio AS, Fagerness J, Crane J, Leboyer M, Delorme R, Pauls DL, et al. Association between polymorphisms in GRIK2 gene and obsessivecompulsive disorder: a family-based study. CNS Neurosci Ther 2011;17: 141-147.

4. Xing J, Kimura H, Wang C, Ishizuka K, Kushima I, Arioka Y, et al. Resequencing and association analysis of six PSD-95-related genes as possible susceptibility genes for schizophrenia and autism spectrum disorders. Sci Rep 2016;6:27491.

5. Lanore F, Labrousse VF, Szabo Z, Normand E, Blanchet C Mulle C. Deficits in morphofunctional maturation of hippocampal mossy fiber synapses in a mouse model of intellectual disability. J Neurosci 2012;32:1788217893.

6. Kim SA, Kim JH, Park M, Cho IH Yoo HJ. Family-based association study between GRIK2 polymorphisms and autism spectrum disorders in the Korean trios. Neurosci Res 2007;58:332-335.

7. Holt R, Barnby G, Maestrini E, Bacchelli E, Brocklebank D, Sousa I, et al. Linkage and candidate gene studies of autism spectrum disorders in European populations. Eur J Hum Genet 2010;18:1013-1019.

8. Dutta S, Das S, Guhathakurta S, Sen B, Sinha S, Chatterjee A, et al. Glutamate receptor 6 gene (GluR6 or GRIK2) polymorphisms in the Indian population: a genetic association study on autism spectrum disorder. Cell Mol Neurobiol 2007;27:1035-1047.

9. Nakanishi M, Nomura J, Ji X, Tamada K, Arai T, Takahashi E, et al. Functional significance of rare neuroligin 1 variants found in autism. PLoS Genet 2017;13:e1006940.

10. Dahlhaus R El-Husseini A. Altered neuroligin expression is involved in social deficits in a mouse model of the fragile X syndrome. Behav Brain Res 2010;208:96-105.

11. Gkogkas CG, Khoutorsky A, Ran I, Rampakakis E, Nevarko T, Weath- erill DB, et al. Autism-related deficits via dysregulated eIF4E-dependent translational control. Nature 2013;493:371-377.

12. Jung M, Haberle BM, Tschaikowsky T, Wittmann MT, Balta EA, Stadler VC, et al. Analysis of the expression pattern of the schizophrenia-risk and intellectual disability gene TCF4 in the developing and adult brain suggests a role in development and plasticity of cortical and hippocampal neurons. Mol Autism 2018;9:20.

13. Pua EPK, Malpas CB, Bowden SC, Seal ML. Different brain networks underlying intelligence in autism spectrum disorders. Hum Brain Mapp 2018;39:3253-3262.

14. Rashid B, Blanken LME, Muetzel RL, Miller R, Damaraju E, Arbabshirani MR, et al. Connectivity dynamics in typical development and its relationship to autistic traits and autism spectrum disorder. Hum Brain Mapp 2018;39:3127-3142.

15. C Yuen RK, Merico D, Bookman M, L Howe J, Thiruvahindrapuram $\mathrm{B}$, Patel RV, et al. Whole genome sequencing resource identifies 18 new candidate genes for autism spectrum disorder. Nat Neurosci 2017;20:602611.

16. Kang HJ, Kawasawa YI, Cheng F, Zhu Y, Xu X, Li M, et al. Spatio-temporal transcriptome of the human brain. Nature 2011;478:483-489.

17. Ginsberg MR, Rubin RA, Natowicz MR. Patterning of regional gene expression in autism: new complexity. Sci Rep 2013;3:1831.

18. Gulsuner S, Walsh T, Watts AC, Lee MK, Thornton AM, Casadei S, et al. Spatial and temporal mapping of de novo mutations in schizophrenia to a fetal prefrontal cortical network. Cell 2013;154:518-529.

19. Yang CP, Li X, Wu Y, Shen Q, Zeng Y, Xiong Q, et al. Comprehensive integrative analyses identify GLT8D1 and CSNK2B as schizophrenia risk genes. Nat Commun 2018;9:838.

20. Choi KH, Zepp ME, Higgs BW, Weickert CS, Webster MJ. Expression profiles of schizophrenia susceptibility genes during human prefrontal cortical development. J Psychiatry Neurosci 2009;34:450-458.

21. Elias GM, Nicoll RA. Synaptic trafficking of glutamate receptors by MAGUK scaffolding proteins. Trends Cell Biol 2007;17:343-352.

22. Carlson GC. Glutamate receptor dysfunction and drug targets across models of autism spectrum disorders. Pharmacol Biochem Behav 2012; 100:850-854.

23. Chez MG, Burton Q, Dowling T, Chang M, Khanna P, Kramer C. Memantine as adjunctive therapy in children diagnosed with autistic spectrum disorders: an observation of initial clinical response and maintenance tolerability. J Child Neurol 2007;22:574-579.

24. Tang J, Yu Y, Yang W. Long noncoding RNA and its contribution to autism spectrum disorders. CNS Neurosci Ther 2017;23:645-656.

25. Strunk D, Weber P, Rothlisberger B, Filges I. Autism and intellectual disability in a patient with two microdeletions in 6q16: a contiguous gene deletion syndrome? Mol Cytogenet 2016;9:88.

26. Jamain S, Betancur C, Quach H, Philippe A, Fellous M, Giros B, et al. Linkage and association of the glutamate receptor 6 gene with autism. Mol Psychiatry 2002;7:302-310.

27. Shuang M, Liu J, Jia MX, Yang JZ, Wu SP, Gong XH, et al. Family-based association study between autism and glutamate receptor 6 gene in Chinese Han trios. Am J Med Genet B Neuropsychiatr Genet 2004;131B:4850.

28. Wang J, Li L, Shao SS, He Z, Chen YL, Kong R, et al. Association analysis of genetic variant of rs13331 in PSD95 gene with autism spectrum disorders: a case-control study in a Chinese population. J Huazhong Univ Sci Technolog Med Sci 2016;36:285-288.

29. Chubykin AA, Atasoy D, Etherton MR, Brose N, Kavalali ET, Gibson JR, et al. Activity-dependent validation of excitatory versus inhibitory synapses by neuroligin-1 versus neuroligin-2. Neuron 2007;54:919-931.

30. Zhang Z, Yu H, Jiang S, Liao J, Lu T, Wang L, et al. Evidence for association of cell adhesion molecules pathway and NLGN1 polymorphisms with schizophrenia in Chinese Han population. PLoS One 2015;10: e0144719.

31. Tristan-Clavijo E, Camacho-Garcia RJ, Robles-Lanuza E, Ruiz A, van der Zee J, Van Broeckhoven C, et al. A truncating mutation in Alzheim- 
er's disease inactivates neuroligin-1 synaptic function. Neurobiol Aging 2015;36:3171-3175.

32. Feng P, Akladious AA, Hu Y. Hippocampal and motor fronto-cortical neuroligin1 is increased in an animal model of depression. Psychiatry Res 2016;243:210-218.

33. Kilaru V, Iyer SV, Almli LM, Stevens JS, Lori A, Jovanovic T, et al. Genome-wide gene-based analysis suggests an association between Neuroligin 1 (NLGN1) and post-traumatic stress disorder. Transl Psychiatry 2016;6:e820.

34. Geschwind DH. Genetics of autism spectrum disorders. Trends Cogn Sci 2011;15:409-416.

35. Klei L, Sanders SJ, Murtha MT, Hus V, Lowe JK, Willsey AJ, et al. Common genetic variants, acting additively, are a major source of risk for autism. Mol Autism 2012;3:9.
36. Tassone F, Qi L, Zhang W, Hansen RL, Pessah IN Hertz-Picciotto I. MAOA, DBH, and SLC6A4 variants in CHARGE: a case-control study of autism spectrum disorders. Autism Res 2011;4:250-261.

37. Wang J, Gong J, Li L, Chen Y, Liu L, Gu H, et al. Neurexin gene family variants as risk factors for autism spectrum disorder. Autism Res 2018; 11:37-43.

38. Tang S, Yao B, Li N, Lin S, Huang Z. Association of dopamine beta-hydroxylase polymorphisms with alzheimer's disease, Parkinson's disease and schizophrenia: evidence based on currently available loci. Cell Physiol Biochem 2018;51:411-428.

39. Suchanek-Raif R, Raif P, Kowalczyk M, Paul-Samojedny M, Kucia K, Merk W, et al. Polymorphic variants of TNFR2 gene in schizophrenia and its interaction with -308G/A TNF-alpha gene polymorphism. Mediators Inflamm 2018;2018:8741249. 